\title{
"Elasticity" variables parameters of nonlinear deformable transversely-isotropic medium
}

\author{
T. D. Karimbayev ${ }^{\dagger}$ \\ †karimbayev@ciam.ru
}

Central Institute of Aviation Motors named after P. I. Baranov, 2 Aviamotornaya st., Moscow, 111116, Russia

Composite materials based on metal and ceramic matrices appear in the design of parts and structures. The mechanical properties of the constituents of these composite materials are comparable. The design of parts made of polymer composite materials is usually based on the assumption of elastic deformations of constituents of the composite material, including the polymer matrix. This assumption does not always work for metal and ceramic composite materials, which makes it difficult to optimize products made of such materials. In addition, the assumption of the elasticity of the composites makes it impossible to estimate the ultimate deformation of the parts. In this connection, nonlinear properties of inhomogeneous, transversally anisotropic composite materials based on metallic or intermetallic matrices are discussed here. It is assumed that the nonlinear properties of composite materials are formed by the nonlinear behavior of the matrix. Earlier, on the basis of the modified theory of plastic flow, nonlinear dependences of the deformation of an anisotropic matrix were obtained. The modified theory of plastic flow makes it possible to take into account the nonlinear dependence not only between deviators of stresses and deformations, but also between the first invariants of stresses and deformations. The nonlinearity in the dependence of the first invariants can be caused by particular manufacturing technology. Five independent strain curves for unidirectionally reinforced composite materials were obtained using the Maxwell and Feucht models. The obtained relations are used to find the stress-strain curves of an aluminum alloy unidirectionally reinforced with boron fibers.

Keywords: composite material, matrix, inhomogeneous, anisotropy, nonlinearity, curves of deforming.

УДК 621.526 .757

\section{Переменные параметры «упругости»}

\section{нелинейно деформируемой трансверсально-изотропной среды}

\author{
Каримбаев Т.Д. ${ }^{\dagger}$
}

Центральный институт авиационного моторостроения им. П.И. Баранова, ул. Авиамоторная, 2, Москва, 111116, Россия

Композиционные материалы на основе металлических и керамических матриц появляются в практике проектирования деталей и узлов силовых конструкций. Механические свойства составляющих этих композиционных материалов сопоставимы. Проектирование деталей из полимерных композиционных материалов обычно основывается на предположении упругих деформаций составляющих композиционного материала, включая полимерную матрицу. Такое предположение далеко не всегда работает для металлических и керамических композиционных материалов, что затрудняет оптимизацию изделий из таких материалов. Кроме того, предположение об упругости составляющих композиционного материала не дает возможности оценить предельные деформации деталей. В этой связи, в настоящей работе обсуждаются нелинейные свойства неоднородных, трансверсально анизотропных композиционных материалов на основе металлических, интерметаллидных и других типов матриц. В работе предполагается, что нелинейные свойства композиционных материалов формируются нелинейным поведением материала матрицы. Ранее на основе модифицированной теории пластического течения были получены нелинейные зависимости деформирования анизотропного материала матрицы. Модифицированная теория пластического течения делает возможным учет нелинейной зависимости не только между девиаторами напряжений и деформаций, но и между первыми инвариантами напряжений и деформаций. Появление нелинейности в зависимости первых инвариантов напряжений и деформаций может быть обусловлено влиянием технологии изготовления изделий на свойства материала матрицы. Пять независимых кривых деформации для однонаправленно армированных композиционных материалов, имеющих анизотропию трансверсально изотропной среды, получены с помощью моделей Максвелла и Фойхта. С помощью полученных соотношений построены и проанализированы кривые зависимости напряжений от деформаций алюминиевого сплава, однонаправленно армированного волокнами бора.

Ключевые слова: композиционный материал, матрица, анизотропия, неоднородность, нелинейность, кривые деформирования. 


\section{1. Введение}

Анизотропная пластичность изучалась, как правило, в связи с перестройкой структуры кристаллической решетки материала после предварительного нелинейного деформирования металлических изделий. Обширная литература, посвященная этой проблеме (см., например, [1], [2]), дополняется не меньшим числом исследований анизотропного нелинейного деформирования кристаллической структуры материалов (см., например, [3], [4]) или горных пород [5]. Однако не так много работ, в которых исследуется нелинейное поведение композиционных материалов (КМ) с различной структурой армирования. В части опубликованных работ (см., например, [6]), как правило, вводятся дополнительные параметры, описывающие нелинейные эффекты, которые подлежат определению из сложных и дорогостоящих испытаний. Другие исследования (см., например, [7]) носят экспериментальный характер, в них приведены кривые деформирования КМ с конкретной схемой армирования и в частных случаях нагружения.

Нелинейное поведение армированных материалов обусловлено нарушением линейной зависимости напряжений от деформаций в одной или нескольких компонентах КМ. Поэтому, прежде всего, следует исследовать неупругое поведение структурных элементов КМ. В подавляющем большинстве современных КМ их неупругое поведение обусловлено нелинейным деформированием материала матрицы, которое может быть описано на основе деформационной теории пластичности [8], теории течения [9] и более общих подходов [10], [11], [12]. При формировании нелинейных физических соотношений материала матрицы могут быть учтены отдельные особенности технологии изготовления КМ $[13,14]$.

Ниже на основе теории течения описан закон деформирования изотропного материала матрицы с учетом возможной нелинейной зависимости гидростатического давления от уровня средней деформации при растяжении. Предложенная зависимость предоставляет возможность аналитически оценить влияние таких технологических несовершенств, как поры, области с излишним или недостаточным содержанием материала матрицы и др. Затем, используя известные зависимости совместного деформирования компонентов КМ [15-18], получены разрешающие уравнения между приращениями напряжений и деформаций для трансверсально-изотропного материала, характеризующегося пятью независимыми кривыми деформирования.

Разработанный подход позволяет аналитически описать сопротивление КМ при сложных путях нагружения.

\section{2. Нелинейное деформирование материала матрицы}

Применительно к металлическим, интерметаллидным КМ модель нелинейного деформирования материала матрицы построена на развитии модели теории течения [9]. Известно, что в компонентах КМ только что изготовленного изделия могут быть начальные напряже- ния и/или деформации, уровень которых определяется используемой технологией изготовления. Кроме того, начальные несовершенства в виде пор, областей с обедненным или повышенным содержанием пор и др. могут оказать влияние на характер деформирования КМ. Это обстоятельство из-за трудностей оценки начальных напряжений и/или деформаций, а также не улавливаемых существующими методами неразрушающего контроля несовершенств, практически не учитывается в известных разработках (см., например, [19]).

\section{1. Соотношения между шаровыми тензорами напряжений и дебормаций}

Предполагается, что объёмная деформация $e=\varepsilon_{i j} \delta_{i j} / 3$ при растяжении может быть нелинейной функцией гидростатического давления $\sigma=\sigma_{i j} \delta_{i j} / 3$. Здесь $\delta_{i j}-$ символ Кронекера, $\varepsilon_{i j}, \sigma_{i j}$ - компоненты тензоров деформаций и напряжений, отсчитываемые от начального состояния $\sigma_{i j}^{0}$, $\varepsilon_{i j}^{0}$; по одинаковым индексам подразумевается суммирование. Нелинейность зависимости $e-\sigma$ материала матрицы КМ обусловлена такими технологическими несовершенствами как несплошность, пористость, рыхлость, инородные включения, области с обогащенным или недостаточным содержанием материала матрицы и их изменения при деформации. Отмеченные дефекты присущи любой известной в настоящее время технологии производства изделий из КМ, в том числе и для изделий, полученных методами аддитивной технологии. Некоторые их типы в большей или меньшей мере присущи отдельным видам технологий.

На участке нелинейных деформаций объёмная деформация е представляется в виде суперпозиции линейных (обратимых) $e^{e}$, температурных $e^{T}$ и нелинейных $e^{n}$ (необратимых) составляющих, записанная в дифференциальной форме

$$
\mathrm{d} e=\mathrm{d} e^{e}+\mathrm{d} e^{n}+\mathrm{d} e^{T} .
$$

Приращения линейных $e^{e}$ и температурных $e^{T}$ деформаций находятся из соотношений

$$
\mathrm{d} e^{e}=\mathrm{d} \sigma / K, \quad \mathrm{~d} e^{T}=\mathrm{d}(\alpha T),
$$

здесь $K$ - объёмный модуль упругости, $\alpha-$ коэффициент линейного расширения. В общем случае объёмные модули упругости при растяжении и сжатии могут различаться $\left(K^{+} \neq K^{-}\right)$.

Приращение нелинейной объёмной деформации $\mathrm{d} e^{n}$ при активном нагружении $\sigma \cdot \mathrm{d} \sigma>0$ определяется из условия $\sigma^{ \pm}(\sigma, \gamma, T)=0$, которое ограничивает предельные значения пропорциональности в зависимости от знака действующего среднего напряжения $\sigma$, накопленной нелинейной средней деформации $\gamma$ и температуры T. Отмеченное условие ниже используется в форме

$$
\sigma^{ \pm}(\sigma, \gamma, T)=\sigma-\sigma^{ \pm}\left(e^{n}, T\right)=0,
$$

Здесь текущие значения пределов пропорциональности гидростатического давления $\sigma$ определяются при растяжении $\sigma^{+}\left(e^{n}, T\right)$ и сжатии $\sigma\left(e^{n}, T\right)$ уровнем накопленной нелинейной средней деформации $e^{n}$ и температуры $T$. Продифференцировав предельное соотношение 
и разрешив результат относительно приращения средней деформации $\mathrm{d} e^{n}$ можно получить

$$
\mathrm{d} e^{n}=\left\{\mathrm{d} \sigma-\left[\frac{\partial\left(\sigma^{n}, T\right)}{\partial T}\right] \cdot \mathrm{d} T\right\} /\left[\frac{\partial \sigma^{ \pm}\left(e^{n}, T\right)}{\partial e^{n}}\right] .
$$

Если температура постоянна $(\mathrm{d} T=0)$, то

$$
\begin{aligned}
& \Psi_{\sigma}=\frac{1}{\left[\frac{\partial \sigma^{ \pm}\left(e^{n}, T\right)}{\partial e^{n}}\right]}=\frac{\mathrm{d} e^{n}}{\mathrm{~d} \sigma^{ \pm}}= \\
& =\frac{\mathrm{d} e}{\mathrm{~d} \sigma^{ \pm}}-\frac{\mathrm{d} e^{e}}{\mathrm{~d} \sigma^{ \pm}}=\frac{1}{K_{\text {tang }}^{ \pm}}-\frac{1}{K} .
\end{aligned}
$$

Параметр $K_{\text {tang }}{ }^{ \pm}$определяется тангенсом угла наклона касательной к экспериментальной поверхности $\sigma=\varphi(e, T)$ в сечении $T=$ const.

Если напряжение постоянно $(\mathrm{d} \sigma=0)$, а температура изменилась на величину $\mathrm{d} T$, то

$$
\Psi_{T}=\frac{\left[\frac{\partial \sigma^{ \pm}\left(e^{n}, T\right)}{\partial T}\right]}{\left[\frac{\partial \sigma^{ \pm}\left(e^{n}, T\right)}{\partial \gamma}\right]}=\frac{\mathrm{d} e^{n}}{\mathrm{~d} T}=\frac{\mathrm{d} e}{\mathrm{~d} T}-\frac{\sigma \mathrm{d} K^{-1}}{\mathrm{~d} T}-\frac{\mathrm{d}(\alpha T)}{\mathrm{d} T} .
$$

Здесь $\mathrm{d} e / \mathrm{d} T$ - тангенс угла наклона касательной к экспериментальной поверхности $\sigma=\varphi(e, T)$ в сечении $\sigma=$ const. Объединяя соотношения (4), (5) можно получить

$$
\begin{array}{cll}
\mathrm{d} e^{n}=0, & \sigma \mathrm{d} \sigma \leq 0 \\
& \text { и } \sigma(\gamma, T) \leq \sigma \leq \sigma^{+}\left(e^{n}, T\right), \\
\mathrm{d} e^{n}=\Psi_{\sigma} \mathrm{d} \sigma+\Psi_{T} \mathrm{~d} T, \quad \text { если } & \sigma \mathrm{d} \sigma>0 \text { и } \sigma<\sigma\left(e^{n}, T\right) \\
& \text { или } \sigma \mathrm{d} \sigma>0 \text { и } \sigma>\sigma^{+}\left(e^{n}, T\right) .
\end{array}
$$

Таким образом, до достижения предельных поверхностей $\sigma^{ \pm}(\gamma, T)$, при разгрузке и нейтральном нагружении объёмная деформация изменяется линейно. При нагрузках, выходящих за пределы текущей предельной поверхности, появляются нелинейные составляющие объёмной деформации $\mathrm{d} e^{n}$ с переменными характеристиками податливости $\Psi_{\sigma}$ и переменными коэффициентами температурного расширения $\Psi_{T}$. Экспериментально построенная поверхность нагружения $\sigma^{ \pm}\left(e^{n}, T\right)$ материала матрицы позволяет определить переменные параметры $\Psi_{\sigma}$ и $\Psi_{T}$.

\section{2. Соотношения между девиаторами напряжений и деформаиий}

Следуя общему способу [9], зависимость компонентов девиатора деформаций $\vartheta_{i j}=\varepsilon_{i j}-e \delta_{i j}$ от компонентов девиатора напряжений $s_{i j}=\sigma_{i j}-\sigma \delta_{i j}$, представляется в виде суперпозиции линейных (обратимых) $\vartheta_{i j}^{e}$ и нелинейных $\vartheta_{i j}^{n}$ составляющих

$$
\ni_{i j}=\vartheta_{i j}^{e}-\ni_{i j}^{n} .
$$

При этом приращения линейных составляющих определяются из

$$
\mathrm{d} \vartheta_{i j}^{e}=\mathrm{d} s_{i j} / 2 G,
$$

где $G$ - модуль сдвига.

В соответствии с теорией течения [9] приращения нелинейных составляющих $\mathrm{d} \vartheta_{i j}^{n}$ деформации считаются пропорциональными компонентам девиатора напряже- ний $s_{i j}$ Здесь коэффициент пропорциональности определяется из обобщенной поверхности $f=0$ Мизеса

$$
f\left(s_{i}, \sigma, T ; \chi_{i}\right)=s_{i}-\sigma_{y}\left(\sigma, T ; \chi_{i}\right)=0 .
$$

Здесь $s_{i}=\left(0.5 s_{i j} s_{i j}\right)^{0.5}-$ интенсивность напряжений, а поверхность нагружения $f=0$ соответствует текущему уровню предела текучести $\sigma_{y}$, который определяется достигнутыми значениями гидростатического давления $\sigma$, температуры $T$ и накопленной нелинейной деформации сдвига $\chi_{i}$. В отличие от изотермической [9] и неизотермической [20] теорий течения здесь предел текучести $\sigma_{y}$ может зависеть ещё от гидростатического давления $\sigma$, позволяя охватить технологические несовершенства материала матрицы.

Используя (8), можно получить

$$
\begin{array}{cr}
\mathrm{d} \vartheta_{i j}^{n}=0, & \text { если } f \leq 0 \text { и } \mathrm{d} f \leq 0, \\
\mathrm{~d} \vartheta_{i j}^{n}=\frac{\left(F^{s} \mathrm{~d} s_{i}-F^{\sigma} \mathrm{d} \sigma-F^{T} \mathrm{~d} T\right) s_{i j}}{s_{i}}, & \text { если } f>0 \text { и } \mathrm{d} f>0 .
\end{array}
$$

Здесь введены обозначения

$$
F^{T}=\left(\frac{3^{0.5}}{3}\right)\left[\frac{\sigma_{11} \mathrm{~d} G^{-1}}{\mathrm{~d} T}-\frac{\mathrm{d} \chi_{i}}{\mathrm{~d} T}\right], \quad \Phi=\left[\frac{1}{G_{\text {tang }}{ }^{2}}-\frac{0.75}{G^{2}}\right]^{0.5},
$$

в которых $G_{\text {tang }}$ - касательная к кривой зависимости интенсивности напряжений $s_{i}$ от интенсивности деформаций $\ni_{i}=\left(0.5 \ni_{i j} \ni_{i j}\right)^{0.5}$ при текущем значении температуры, $\mathrm{d} \chi_{i} / \mathrm{d} T-$ касательная к кривой зависимости интенсивности деформаций $э_{i}$ от температуры при текущем значении напряжения $s_{i}$.

Таким образом, до достижения предельной поверхности $f=0$, при разгрузке $\mathrm{d} f<0$ и нейтральном нагружении $\mathrm{d} f=0$ компоненты девиатора деформаций изменяются линейно. При нагрузках, выходящих за пределы текущей предельной поверхности $f>0$ и $\mathrm{d} f>0$, появляются нелинейные составляющие девиатора деформаций $\mathrm{d} \vartheta_{i j}^{n}$ с переменными характеристиками податливости $F^{s}$ и температурными эффектами $F^{T}$. Экспериментально построенная поверхность нагружения $\sigma_{y}\left(\sigma, T ; \chi_{i}\right)$ материала матрицы позволяет определить параметры $F^{s}$ и $F^{T}$.

\section{3. Зависимости дебормаций от напряжений}

Зависимости деформаций от напряжений для материала матрицы принимают общую форму

$$
\mathrm{d} \varepsilon_{i j}=a_{i j m n} \mathrm{~d} \sigma_{i j}+\Omega_{i j} \mathrm{~d} T .
$$

Переменные параметры податливости $a_{i j m n}$ и приведенные коэффициенты линейного расширения целесообразно записать с применением технических характеристик [21]. В этом случае

$$
\begin{aligned}
& a_{i i i i}=\frac{1}{E_{i i}}=\frac{1}{E}+\frac{\left[\Psi_{\sigma}-\left(\frac{s_{i i}}{s_{i}}\right) F^{T}\right]}{3}+F^{s} \frac{\left(\frac{s_{i i}}{s_{i}}\right)^{2}}{2} \\
& a_{i i j j}=\frac{v_{i j}}{E_{i i}}=-\frac{v}{E}+\frac{\left[\Psi_{\sigma}-\left(\frac{s_{i i}}{s_{i}}\right) F^{T}\right]}{3}+F^{s} \frac{\left(\frac{s_{i i}}{s_{i}}\right)}{2 s_{i}^{2}}, \quad j \neq i \\
& a_{i i m n}=\frac{\eta_{m n, i}}{E_{i i}}=F^{s} \frac{\left(\frac{s_{i i}}{s_{m n}}\right)}{2 s_{i}^{2}}, \quad m \neq n
\end{aligned}
$$




$$
\begin{aligned}
& \Omega_{i i}=\alpha+\frac{T \mathrm{~d} \alpha}{\mathrm{d} T}+\Psi_{T}+\left(\frac{s_{i i}}{2}\right) \frac{\mathrm{d} G^{-1}}{\mathrm{~d} T}-F^{s}\left(\frac{s_{i i}}{s_{i}}\right) \\
& a_{i j i j}=\frac{1}{2 G_{i j}}=\frac{1}{2 G}+F^{s}\left(\frac{s_{i j}}{s_{i}}\right)^{2}, i \neq j \\
& a_{m n i i}=\frac{\eta_{i, m n}}{2 G_{m n}}=\left(\frac{s_{m n}}{s_{i}}\right)\left[\left(\frac{s_{i i}}{2 s_{i}}\right) F^{s}-F^{\sigma}\right], \quad m \neq n \\
& a_{i j m n}=\frac{\mu_{i j, m n}}{2 G_{m n}}=F^{s}\left(\frac{s_{i j}}{s_{i}}\right)\left(\frac{s_{m n}}{s_{i}}\right), \quad j \neq i, m \neq n, j \neq n \\
& \Omega_{i j}=\left(\frac{s_{i j}}{2}\right)\left(\frac{\mathrm{d} G^{-1}}{\mathrm{~d} T}\right)-F^{T}\left(\frac{s_{i j}}{s_{i}}\right), \quad j \neq i
\end{aligned}
$$

Здесь $E_{i i}$ - переменные модули упругости на растяжение, сжатие вдоль осей $x_{i}(i=1,2,3) ; v_{i j}-$ коэффициенты Пуассона, характеризующие сжатие в направлении первого индекса при растяжении в направлении второго индекса; $\eta_{m n, i}$ - коэффициенты влияния первого рода, характеризующие сдвиги в плоскости $x_{m} x_{n}$ при растяжении в направлении $x_{i} ; G_{i j}-$ модули сдвига в плоскости $x_{i} x_{j}$; $\eta_{i, m n}-$ коэффициенты взаимного влияния второго рода, характеризующие растяжение (сжатие) в направлении оси $x_{i}$ при сдвиге в плоскости $x_{m} x_{n} ; \mu_{i j, m n}-$ коэффициенты Ченцова, характеризующие сдвиги в плоскости $x_{i} x_{j}$ при сдвиге в плоскости $x_{m} x_{n}$.

\section{3. Переменные параметры «упругости» нелинейного деформирования трансверсально-изотропных КМ}

Предполагается, что физические соотношения нелинейно деформируемой трансверсально-анизотропной среды в приращениях можно представить в виде

$$
\begin{aligned}
& \mathrm{d} \varepsilon_{11}=\frac{\left(\mathrm{d} \sigma_{11}-v_{12} \mathrm{~d} \sigma_{22}-v_{12} \mathrm{~d} \sigma_{33}\right)}{E_{1}}+\alpha_{1} \mathrm{~d} T, \\
& \mathrm{~d} \varepsilon_{12}=\frac{\mathrm{d} \sigma_{12}}{G_{12}}, \\
& \mathrm{~d} \varepsilon_{22}=\frac{\left(-v_{21} \mathrm{~d} \sigma_{11}+\mathrm{d} \sigma_{22}-v_{23} \mathrm{~d} \sigma_{33}\right)}{E_{2}}+\alpha_{2} \mathrm{~d} T, \\
& \mathrm{~d} \varepsilon_{13}=\frac{\mathrm{d} \sigma_{13}}{G_{12}}, \\
& \mathrm{~d} \varepsilon_{33}=\frac{\left(-v_{21} \mathrm{~d} \sigma_{11}-v_{23} \mathrm{~d} \sigma_{22}+\mathrm{d} \sigma_{33}\right)}{E_{3}}+\alpha_{3} \mathrm{~d} T, \\
& \mathrm{~d} \varepsilon_{23}=\frac{\mathrm{d} \sigma_{23}}{G_{23}},
\end{aligned}
$$

с пятью независимыми переменными параметрами «упругости» $E_{1}, E_{2}, v_{12}, v_{23}, G_{12}$ и двумя переменными теплофизическими характеристиками $\alpha_{1}, \alpha_{2}$. При этом имеют место известные соотношения $v_{12} / E_{1}=v_{21} / E_{2}$, $G_{23}=E_{2} /\left(1+v_{23}\right)$.

Аналитическое определение параметров упругости трансверсально-изотропного тела приведено во многих работах (см., например, [15], [16], [17], [18]). В известных аналитических подходах не учитываются особенности материала матрицы, обусловленные технологией их переработки при изготовлении КМ, а также возможные нелинейные деформации. Однако основополагающие предположения в этих подходах могут быть использованы и используются здесь.

\section{1. Растяжение вдоль оси изотропии}

Пусть трансверсально-изотропная неоднородная среда находится под действием нагрузки, направленной вдоль оси изотропии $\sigma_{11}=\sigma$ и дополнительная нагрузка $\mathrm{d} \sigma$ направлена также в этом направлении. В соответствии с (13) в среде

$$
\mathrm{d} \varepsilon_{11}=\frac{\mathrm{d} \sigma_{11}}{E_{1}}, \quad \mathrm{~d} \varepsilon_{22}=\mathrm{d} \varepsilon_{33}=-\frac{v_{21} \mathrm{~d} \sigma_{11}}{E_{2}}=-v_{12} \mathrm{~d} \varepsilon_{11} .
$$

Остальные компоненты напряженно-деформированного состояния равны нулю. При этом в компонентах КМ ( $\alpha=1$ волокна, $\alpha=2$ матрица) реализуется напряженно-деформированное состояние, характеризуемое приращениями

$$
\mathrm{d} \varepsilon_{11}=\mathrm{d} \varepsilon_{11}^{\alpha}=\frac{\mathrm{d} \sigma_{11}^{\alpha}}{E_{11}^{\alpha}}, \quad \mathrm{d} \varepsilon_{22}^{\alpha}=\mathrm{d} \varepsilon_{33}^{\alpha}=-v_{12} \mathrm{~d} \varepsilon_{11}^{\alpha}=-v_{12}^{\alpha} \mathrm{d} \varepsilon_{11} .
$$

Приращение напряжений в составном материале является суперпозицией приращений напряжений в компонентах КМ с коэффициентами пропорциональности, равными объёмному $\mathrm{v}^{\alpha}$ содержанию компонентов. Использование этого правила приводит к правилу «смесей»

$$
E_{1}=\mathrm{v}^{1} E_{11}^{1}+\mathrm{v}^{2} E_{11}^{2}, \quad v_{12}=\mathrm{v}^{1} v_{12}^{1}+\mathrm{v}^{2} v_{12}^{2},
$$

в которых вышеприведенными соотношениями (см. раздел 2) учтены нелинейные особенности материала матрицы.

\section{2. Растяжение в плоскости изотропии}

Пусть образец растягивается в плоскости изотропии. Для определенности он растягивается в направлении оси $x_{2}$ и достигнутое напряженное состояние характеризуется напряжением $\sigma_{22}=\sigma$. Дополнительное напряжение $\mathrm{d} \sigma$ в том же направлении в соответствии с соотношениями (13) приводит к деформациям КМ

$$
\mathrm{d} \varepsilon_{11}=-\frac{v_{12} \mathrm{~d} \sigma}{E_{1}}, \quad \mathrm{~d} \varepsilon_{22}=\frac{\mathrm{d} \sigma}{E_{2}}, \quad \mathrm{~d} \varepsilon_{33}=-\frac{v_{23} \mathrm{~d} \sigma}{E_{2}} .
$$

В компонентах КМ при растяжении в плоскости изотропии могут появиться осевые напряжения $\mathrm{d} \sigma_{11}^{\alpha}$. При этом предполагается, что

- приращения осевых деформаций в компонентах композиции и в КМ одинаковы, т. е. $\mathrm{d} \varepsilon_{11}^{\alpha}=\mathrm{d} \varepsilon_{11}$,

- приращения деформаций $\mathrm{d} \varepsilon_{33}^{\alpha}$ в плоскости изотропии определятся из равенства $\mathrm{d} \varepsilon^{\alpha}{ }_{33}=-v_{23}^{\alpha} \mathrm{d}_{22}^{\alpha}$

- приращения деформаций $\mathrm{d} \varepsilon_{22}, \mathrm{~d} \varepsilon_{33}$ КМ в плоскости изотропии определяются суперпозицией приращений соответствующих деформаций в компонентах КМ с коэффициентами пропорциональности, равными объёмному ${ }^{\alpha}(\alpha=1,2)$ содержанию компонентов $\mathrm{d} \varepsilon_{22}=\mathrm{v}^{1} \mathrm{~d} \varepsilon_{22}^{1}+\mathrm{v}^{2} \mathrm{~d} \varepsilon_{22}^{2}, \mathrm{~d} \varepsilon_{33}=\mathrm{v}^{1} \mathrm{~d} \varepsilon_{33}^{1}+\mathrm{v}^{2} \mathrm{~d} \varepsilon_{33}^{2}$.

B рамках этих предположений приращения осевых напряжений $\mathrm{d} \sigma_{11}^{\alpha}$ в компонентах определяются из равенств

$$
\mathrm{d} \sigma_{11}^{\alpha}=\left(v_{12}^{\alpha}-\frac{v_{12} E_{11}^{\alpha}}{E_{1}}\right) \mathrm{d} \sigma
$$


Здесь $E_{1}$ и $v_{12}$ характеристики КМ, определенные из равенств (14). В результате получаются соотношения для модуля упругости $E_{2}$ и коэффициента Пуассона $v_{23}$ в плоскости изотропии

$$
\begin{aligned}
& E_{2}^{-1}=\frac{\mathrm{v}^{1}\left[1-v_{21}^{1}\left(v_{12}^{1}-\frac{v_{12} E_{11}^{1}}{E_{1}}\right)\right]}{E_{22}^{1}}+\frac{\mathrm{v}^{2}\left[1-v_{21}^{2}\left(v_{12}^{2}-\frac{v_{12} E_{11}^{2}}{E_{1}}\right)\right]}{E_{22}^{2}},(15) \\
& v_{23}=\frac{\mathrm{v}^{1}\left[v_{23}^{1}+v_{21}^{1}\left(v_{12}^{1}-\frac{v_{12} E_{11}^{1}}{E_{1}}\right)\right]}{E_{22}^{1}}+\frac{\mathrm{v}^{2}\left[v_{23}^{2}+v_{21}^{2}\left(v_{12}^{2}-\frac{v_{12} E_{11}^{2}}{E_{1}}\right)\right]}{E_{22}^{2}} \cdot E_{2} .
\end{aligned}
$$

\section{3. Сдвиг в плоскости армирования}

Пусть при испытаниях на сдвиг в плоскости армирования $x_{1} x_{2}$ образец нагружен до напряжений $\sigma_{12}=\tau$ и он получил дополнительное нагружение $\mathrm{d} \sigma_{12}=\mathrm{d} \tau$. В этом случае в КМ в соответствии с (13) появятся дополнительные сдвиговые деформации $\mathrm{d} \varepsilon_{12}=\mathrm{d} \tau / G_{12}$. Предполагается, что все компоненты тензоров напряжений и деформаций, как в КМ, так и его компонентах пренебрежимо малы по сравнению со сдвигом. Приращения сдвиговых деформаций $\mathrm{d} \varepsilon_{12}$ в плоскости армирования определяются суперпозицией приращений соответствующих деформаций в компонентах КМ с коэффициентами пропорциональности, равными объёмному $\mathrm{v}^{\alpha}(\alpha=1,2)$ содержанию компонентов $\mathrm{d} \varepsilon_{12}=\mathrm{v}^{1} \mathrm{~d} \varepsilon_{12}^{1}+\mathrm{v}^{2} \mathrm{~d} \varepsilon_{12}^{2}$.

$\mathrm{B}$ рамках этих предположений можно получить, что

$$
\frac{1}{G_{12}}=\frac{\mathrm{v}^{1}}{G_{12}^{1}}+\frac{\mathrm{v}^{2}}{G_{12}^{2}},
$$

что завершает определение пяти независимых переменных параметров упругости КМ.

В соотношениях (14) - (16) характеристики матрицы $(\alpha=2)$ определяются из равенств (12) при соответствующих напряжениях и деформациях, определяя переменность параметров «упругости» в зависимостях (13).

\section{4. Расчетные кривые зависимости для однонаправлено-армированного боралюминия}

Ниже с использованием разработанной расчетной программы построены пять независимых кривых деформирования для однонаправлено-армированного боралюминия. В качестве матрицы использовался сплав АД33 со свойствами из [22]. Свойства волокон бора приведены в Табл. 1.

Табл. 1. Упругие свойства борных волокон.

Table 1. Elastic properties of boron fibers.

\begin{tabular}{|c|c|c|c|c|c|}
\hline $\begin{array}{c}E, \Gamma \Pi \mathrm{a} \\
E, \mathrm{GPa}\end{array}$ & $\begin{array}{c}G, \text { ГПа } \\
G, \mathrm{GPa}\end{array}$ & $v$ & $\begin{array}{c}\sigma, \mathrm{M \Pi а} \\
\sigma, \mathrm{MPa}\end{array}$ & $\delta, \%$ & $\alpha 10^{6}, 1 / \mathrm{C}^{\circ}$ \\
\hline 385 & 175 & 0.1 & 3150 & 0.8 & 7.0 \\
\hline
\end{tabular}

Изделия из боралюминия изготавливают горячим прессованием заготовок, полученных выкладкой листов однонаправлено уложенных борных волокон, напыленных алюминием. Рассеяние свойств изделия обусловлено как технологией переработки, так и состоянием заготовки. В расчетах в качестве исходных данных использовались кривые деформирования алюминиевого сплава АД33, и моделированная нами нелинейная часть зависимости гидростатического давления от средней деформации (см. Рис. 1).

Расчетные кривые деформирования КМ (боралюминия) с различным объёмным содержанием борных волокон приведены на Рис. 2-4. Здесь объёмное содержание волокна $\mathrm{v}^{1}$ изменялось от 0.2 с шагом 0.15 до 0.65 . Можно видеть, что с увеличением объёмного содержания волокон несущая способность КМ возрастает. Предельные деформации КМ при растяжении вдоль волокон (Рис. 2a) определяются предельными деформациями волокна и влияние свойств материала матрицы, в том числе нелинейные, незначительно сказываются на зависимости $\sigma_{11} \sim \varepsilon_{11}$ КМ. При продольном растяжении вдоль волокон эффект нелинейных деформаций материала матрицы заметно сказывается на поперечных деформациях $\varepsilon_{22}$ (см. Рис. 2b). Пределы пропорциональности попереч-
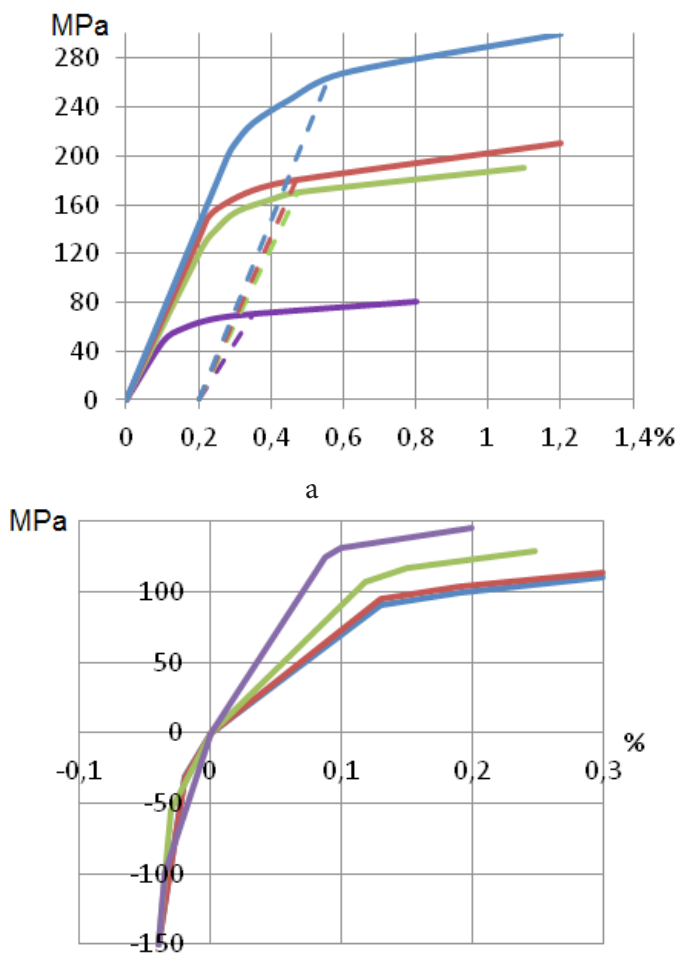

$\mathrm{b}$

Puc. 1. (Color online) Зависимости девиаторов (а) и средних напряжений (b) от деформации алюминиевого сплава АД-33 при различных температурах $\left(20^{\circ} \mathrm{C}-\right.$ синяя линия, $150^{\circ} \mathrm{C}$ - красная, $200^{\circ} \mathrm{C}$ - светлозеленая, $300^{\circ} \mathrm{C}$ - фиолетовая).

Fig. 1. (Color online) Dependence of deviators (a) and mean stresses (b) on the deformation of aluminum alloy AD-33 at different temperatures $\left(20^{\circ} \mathrm{C}-\right.$ blue line, $150^{\circ} \mathrm{C}-$ red line, $200^{\circ} \mathrm{C}$ - green line, $300^{\circ} \mathrm{C}$ - violet line). 
ных деформаций имеют место при одинаковых уровнях для КМ с различным объёмным содержанием волокон.

Наиболее ярко нелинейности КМ проявляются при их деформировании в плоскости изотропии. На Рис. За приведена зависимость $\sigma_{22} \sim \varepsilon_{22}$ при растяжении КМ в плоскости изотропии. Характерно, что с увеличением объёмного содержания волокон $\mathrm{v}^{1}$ предельные деформации $\varepsilon_{22}$ заметно снижаются. При малом объёмном содержании волокон $\left(\mathrm{v}^{1}=0.2\right)$ предельная деформация КМ мало отличается от предельной деформации материала матрицы.

На Рис. 3b приведена зависимость степени сжатия КМ $\varepsilon_{32}$ при растяжении в плоскости изотропии $\sigma_{22} \sim \varepsilon_{23}$.

Последней (пятой) независимой характеристикой однонаправлено армированного КМ является характеристика сдвига $\sigma_{12} \sim \varepsilon_{12}$ в плоскости армирования. Расчетные нелинейные зависимости $\sigma_{12} \sim \varepsilon_{12}$ КМ приведены на Рис. 4. Также как и в предыдущем случае, уровни предельных деформаций зависят от объёмного содержания волокна.
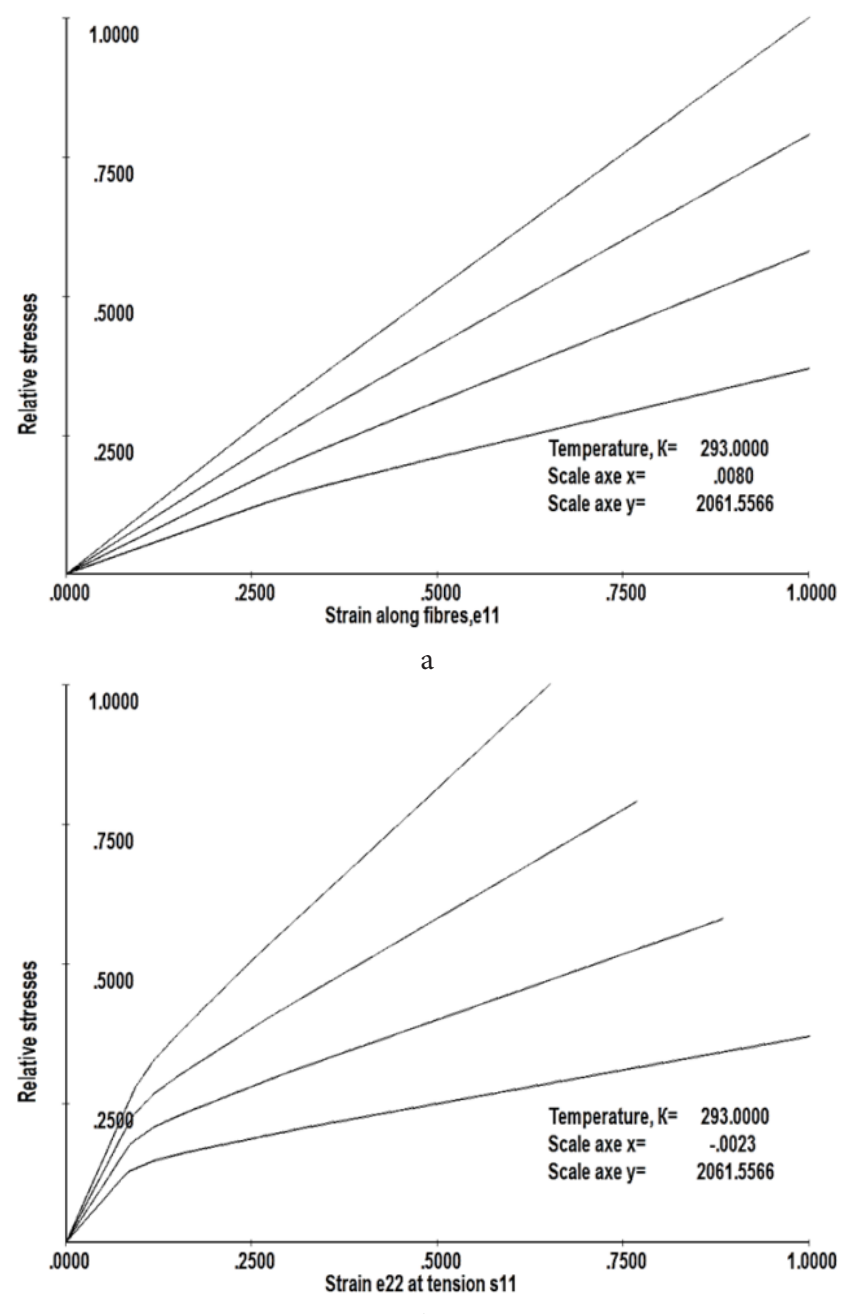

$\mathrm{b}$

Рис. 2 Кривые деформирования однонаправленоармированного КМ при растяжении вдоль волокон: продольные деформации (a), поперечные деформации (b).

Fig. 2. Stress-strain curves of unidirectional reinforced composite materials at tension along the fibres: longitudinal strain (a), transverse strain (b).

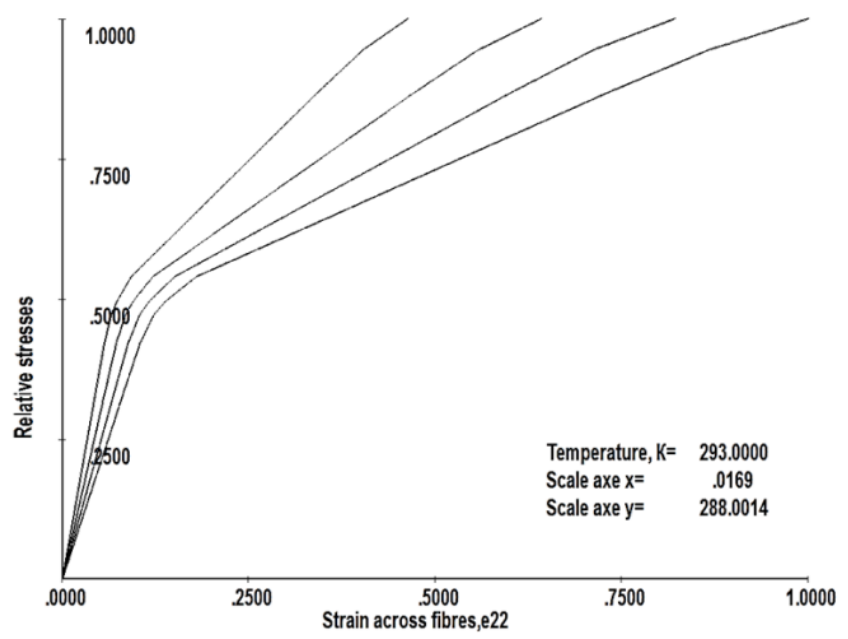

a

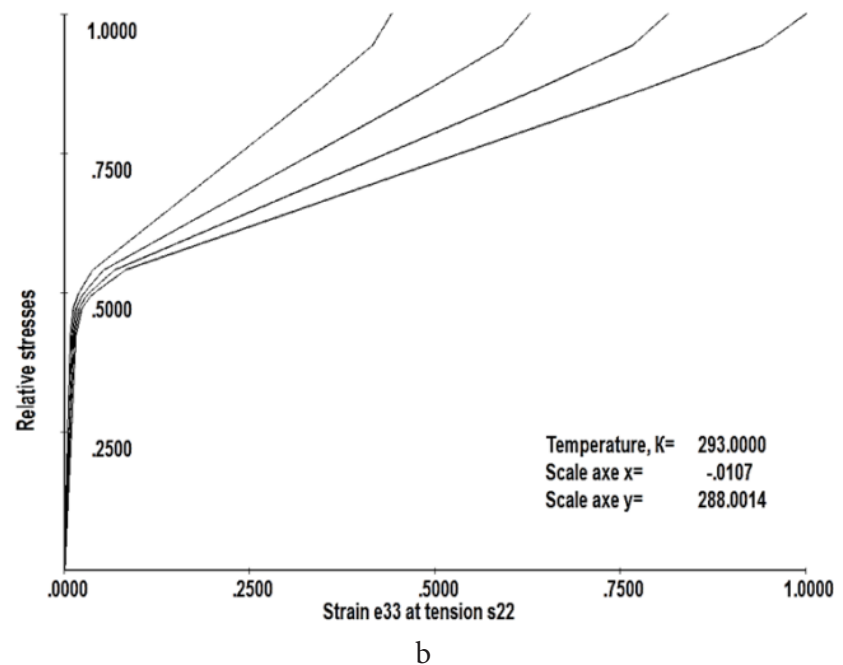

Рис. 3. Кривые деформирования однонаправленоармированного КМ при растяжении в плоскости изотропии: продольные деформации (a), поперечные деформации (b).

Fig. 3. Stress-strain curves of unidirectional reinforced composite material at tension in the plane of isotropy: longitudinal strain (a), transverse strain (b).

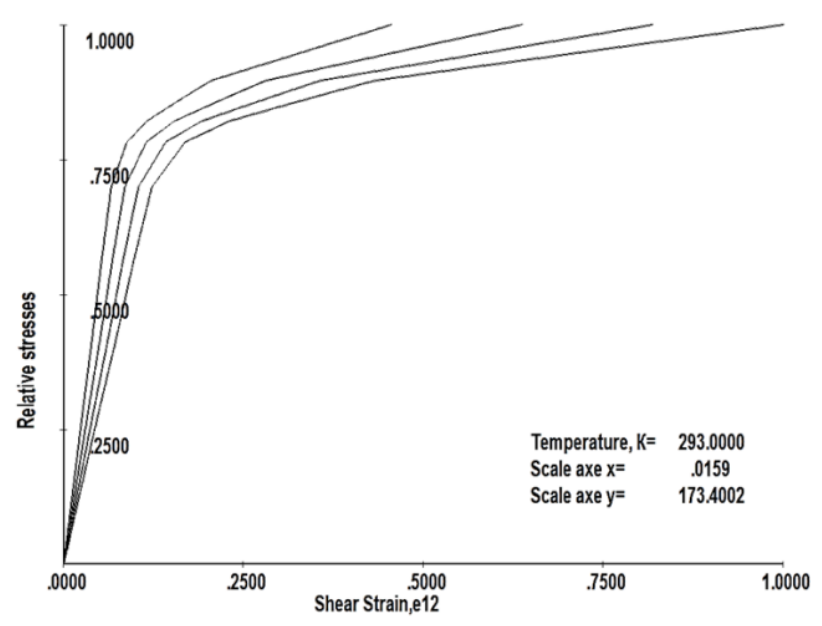

Рис. 4. Кривые деформирования однонаправленоармированного КМ при сдвиге в плоскости армирования.

Fig. 4. Stres-strain curves of unidirectional reinforced composite material at shear in plane reinforcement. 


\section{5. Заключение}

Разработана методика предсказания нелинейного поведения однонаправленно армированных КМ на основе нелинейного деформирования материала матрицы. Нелинейное деформирование материала матрицы описано модифицированными соотношениями теории течения, позволяющими учесть технологические дефекты. Разработанный подход позволяет исследовать сопротивление однонаправленно армированных КМ при сложных путях нагружения.

\section{Литература/References}

1. P. G.Kiklyaev, Ya. B. Fridman. Anisotropy of mechanical properties of metals. Moscow, Metallurgy (1986) 223 p. (in Russian) [П.Г. Микляев, Я.Б. Фридман. Анизотропия механических свойств металлов. Москва, Металлургия (1986) 223 с.]

2. S.A. Berestova. Deformation anisotropy volumeisotropic structurally heterogeneous media: dissertacija na soiskanie stepeni doctora fiziko-matematicheskih nauk. Yekaterinburg (2006) 349 p. (in Russian) [C.А. Берестова. Деформационная анизотропия объёмно-изотропных структурно-неоднородных сред: диссертация на соискание ученой степени доктора физ-мат наук. Екатеринбург (2006) 349 с.]

3. A.I. Chanyshev. Applied Mechanics and Technical Physics. 2, 149 (1984). (in Russian) [А.И. Чанышев. Прикл. механика и техн. физика. 2, 149 (1984) .]

4. Ya. A. Erisov, F. V. Grechenkov, S. B. Surudin. Bulletin of MSTU G.I. Nosova. 14(4), 42 (2016). (in Russian) [Я. А. Ерисов, Ф. В. Греченков, С. В. Сурудин. Вестник МГТУ им. Г. И. Носова. 14(4), 42 (2016).]

5. L.L. Efimenko. Construction of elastoplastic models for anisotropic media: dissertacija na soiskanie stepeni kandidata fiziko-matematicheskih nauk. Novosibirsk (2007) 140 p. (in Russian) [Л. Л. Ефименко. Построение упругопластических моделей для анизотропных сред: диссертация на соискание ученой степени кандидата физ-мат наук. Новосибирск (2007) 140 с.]

6. O. V. Salov. Nonlinear deformation of two-matrix composite structures: aftoreferat dissertacii na soiskanie stepeni kandidata tehnicheskih nauk. Moscow (1999) 14 p. (in Russian) [О.В. Салов. Нелинейное деформирование двухматричных композитных структур: автореферат диссертации на соискание ученой степени кандидата технических наук. Москва (1999) 14 с.]

7. L. Zawada, G. Ojard, E. Bouillon, P. Spriet, C. Legan. Evalution of Ceramic Matrix Composite Exhaust Nozzle Divergent Seals. Proceedings of the 43-th AIAA/ASME/SAE/ASEE Joint Conference and Exhibit. Cincinati (2007) pp. AIAA 2007-5082. DOI: $10.2514 / 6.2007-5082$
8. A. A. Ilyushin. Plasticity. Moscow, Gostekhizdat (1948) 348 p. (in Russian) [А.А. Ильюшин. Пластичность. Москва, Гостехиздат (1948) 348 с.]

9. L. M. Kachanov. Fundamentals of the Theory of Plasticity. Moscow, Nauka (1969) 420 p. (in Russian) [Л. М. Качанов. Основы теории пластичности. Москва, Наука (1969) 420 c.]

10. A. A. Ilyushin. Continuum mechanics. Moscow, MGU (1978) 287 p. (in Russian) [А.А. Ильюшин. Механика сплошной среды. Москва, МГУ (1978) 287 с.]

11. V.V. Novozhilov. Problems of Continuum Mechanics. Leningrad, Sudostroenie (1989) 400 p. (in Russian) [B.В. Новожилов. Вопросы механики сплошной среды. Ленинград, Судостроение (1989) 400 с.]

12. B. D. Annin, V.M. Zhigalkin. Behavior of materials under conditions of complex loading. Novosibirsk, Siberian Branch of the RAS (1999) 341 p. (in Russian) [Б.Д. Аннин, В.М. Жигалкин. Поведение материалов в условиях сложного нагружения. Новосибирск, CO PAH (1999) 341 c.]

13. T.D. Karimbaev. Trudy CIAM. 1109(3), 93 (1985). (in Russian) [Т.Д. Каримбаев. Труды ЦИАМ. 1109(3), 93 (1985).]

14. T.D. Karimbaev, B. Myktybekov, I. M. Panova. Trudy CIAM. 1334, 160 (2005). (in Russian) [Т. Д. Каримбаев, Б. Мыктыбеков, И.М. Панова. Труды ЦИАМ. 1334, 160 (2005).]

15. D. S. Abolinysh. Mechanics of polymers. 4, 52 (1965). (in Russian) [Д. С. Аболиньш. Механика полимеров. 4, 52 (1965).]

16. V. V. Bolotin. Calculations on the strength. 12, 3 (1966). (in Russian) [В.В. Болотин. Расчеты на прочность. 12, 3 (1966).]

17. S. W. Tsai. Aeronutronic Publication. U-1699 (1962).

18. R. Christensen. An Introduction to Composite Mechanics. Moscow, Mir (1982) 334 p. (in Russian) [Р. Кристенсен. Введение в механику композитов. Москва, Мир (1982) 334 c.]

19. B. E. Pobedrya. Applied Mathematics and Mechanics. 48 (4), 29 (1984). (in Russian) [Б. Е. Победря. Прикладная математика и механика. 48 (4), 29 (1984).]

20. Yu.N. Shevchenko. Thermoplasticity under variable loading. Kiev, Naukova Dumka (1979) 287 p. (in Russian) [Ю.Н. Шевченко. Термопластичность при переменных нагружениях. Киев, Наукова Думка (1979) 287 с.]

21. S. G. Lekhnitsky. Theory of Elasticity of an Anisotropic Body. Moscow, Nauka (1977) 415 p. (in Russian) [C.Г. Лехницкий. Теория упругости анизотропного тела. Москва, Наука (1977) 415 с.]

22. Ed. A. T. Tumanov. Aviation materials: Handbook in 9 volumes, V. 4, Part 1. Moscow, ONTI (1982) 625 p. (in Russian) [Под общ. ред. А. Т. Туманова. Авиационные материалы: Справочник в 9 томах, Том 4, Часть 1. Москва, ОНТИ (1982) 625 с.] 